\title{
Linac booster for high energy proton therapy and imaging
}

\author{
Alberto Degiovanni* and Ugo Amaldi \\ TERA Foundation, 28100 Novara, Italy \\ Anthony J. Lomax, Jacobus M. Schippers, and Lukas Stingelin \\ Paul Scherrer Institute, 5232 Villigen-PSI, Switzerland \\ Javier Bilbao de Mendizabal ${ }^{\dagger}$ \\ Université de Genève, CH-1211 Genève 4, Switzerland
}

(Received 9 October 2017; published 28 June 2018)

\begin{abstract}
For an optimal exploitation of the benefits of proton therapy the most accurate dose delivery system should be used. The TERA Foundation has extensive experience in the field of high gradient high frequency linacs. This paper describes a particular design of a $3 \mathrm{GHz}$ linac boosting the typical cyclotron beams for proton therapy of $230-250 \mathrm{MeV}$ up to $350 \mathrm{MeV}$. Such an upgrade of a typical proton therapy facility enables performing proton radiography, as well as extending therapeutic capabilities with high energy proton therapy (HEPT). The recent studies and measurements in high-gradient linac technology demonstrated that average fields in the accelerating structures of up to $25-30 \mathrm{MV} / \mathrm{m}$ can be achieved, which results in a total linac length of less than $7 \mathrm{~m}$. To test several characteristics of such a linac as a booster of a cyclotron beam, a design has been made of a linac unit accelerating from $250 \mathrm{MeV}$ to $275 \mathrm{MeV}$, which could be built and inserted for tests in an existing beam line at the PSI proton therapy facility. The feasibility considerations, along with the design of the linac booster and the issues related to a possible integration in an existing cyclotron beam line are detailed in this study.
\end{abstract}

DOI: 10.1103/PhysRevAccelBeams.21.064701

\section{INTRODUCTION}

The number of cancer patients treated with proton therapy is increasing exponentially. The demand for proton therapy treatment facilities is also growing very fast. Although many facilities are based on traditional dose delivery methods (passive scattering), a rapidly growing number are based on the more state-of-the-art pencil beam scanning (PBS) approach [1,2].

Nevertheless, PBS proton therapy technology is not yet fully mature, offering therefore a big potential for new developments addressing current limitations, including the management of organ motion, the need for improved lateral penumbras and last but not least, the actual value of in-vivo proton stopping power. To overcome these limitations, novel approaches are under study in the medical physics

\footnotetext{
*alberto.degiovanni@cern.ch

Present address: ADAM SA, 1217 Meyrin, Switzerland.

Present address: MELEXIS TECHNOLOGIES SA, 2022 Bevaix, Switzerland.

Published by the American Physical Society under the terms of the Creative Commons Attribution 4.0 International license. Further distribution of this work must maintain attribution to the author(s) and the published article's title, journal citation, and DOI.
}

community. These include magnetic resonance imaging (MRI) guided proton therapy, proton imaging, proton tomography, and high energy proton therapy (HEPT) [3].

In particular, these last two applications require proton beams at higher energies than the conventionally 230$250 \mathrm{MeV}$ available in today's treatment facilities. Proton beams with an energy of $350 \mathrm{MeV}$ - equivalent to a range of more than $60 \mathrm{~cm}$ in water equivalent tissue (WET) would fully traverse the body of the patient, allowing new treatment methods. The availability of such high energies in existing and new treatment facilities will open a spectrum of new possibilities for proton therapy (Fig. 1). In an existing facility, this higher proton energy can be achieved by boosting the current energy of the clinical proton beams. We are proposing to use such an upgrade for the following three applications: proton radiography, the treatment of very small tumors and the treatment of tumors that are closely surrounded by critical organs [4]. Indeed, the higher energy protons would traverse the patient body at all possible treatment sites. These traversing protons can be used for proton radiography. Furthermore, the higher energy proton beams will have a smaller lateral penumbra compared to current proton beams and comparable to the one obtained with carbon ions. This small beam profile can be used to sharpen the proton dose distribution at critical boundaries of the tumor volume, such as the ones near a 

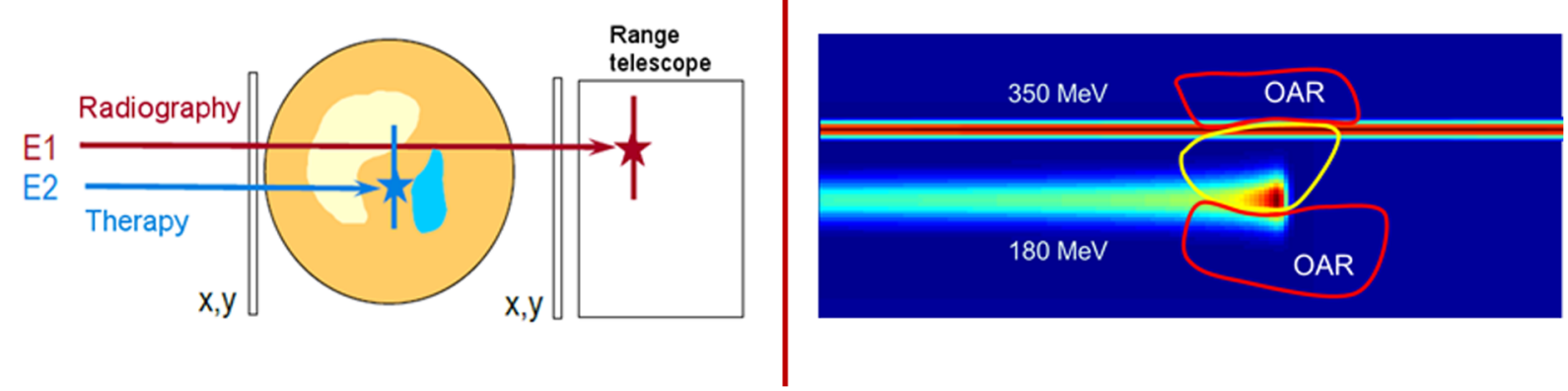

FIG. 1. Left: In a schematic presentation of proton radiography it is shown how the remaining energy of protons with initial energy E1 is measured by means of a range telescope. In this way, the total stopping power along the proton track in the patient is measured. This information is used for a very accurate calculation of the range of lower energy protons (E2) used in therapy. Right: A tumour irradiation with Bragg peaks will also give a dose next to the tumour due to multiple scattering. By using high energy, when irradiating the lateral edge of the tumor, the lateral penumbra is decreased substantially. Behind the tumor only a small volume is receiving extra dose due to the longer range of high energy protons.

critical healthy structure close to the tumor or it can be used for the treatment of very small tumors. In particular, the high-energy beams are to be used at the lateral target edges only. Due to the lower multiple scattering at high energy, this will sharpen the lateral penumbra in the direction transverse to the high-E pencil beam. This penumbra with a steeper dose gradient is usually considered a major advantage [5]. The dose distal to the target due to these few high-E pencil beams, will only occur in a very small volume.

The study in the IMPULSE project, initiated by the Paul Scherer Institute in 2011 [6], aims at addressing the abovementioned challenges to develop new technologies which can improve the benefits of proton therapy.

This paper describes the possibility of using a linac for the IMPULSE project, starting with its general layout, which resembles the cyclinac design [7]. This is followed by a description of a specific design including the optimization steps of the rf design and the results of the beam dynamics studies. In particular it is discussed how the new rf design can create the relatively strong electric fields needed to obtain the desired final energy within a relatively short length of the linac. At the end, a test program is proposed taking into consideration the integration within existing facilities.

\section{DESCRIPTION OF THE PROJECT}

Among the many plausible technological approaches to achieve higher energies and, considering the medical accelerator solutions developed since 15 years by the TERA Foundation [7], the use of a linac booster for HEPT seems to be a relatively easy-to-achieve major improvement.

The design of the IMPULSE linac builds on the experience gained from the design and construction of the LIBO (LInac BOoster) prototype in 1998 (Fig. 2), in collaboration with CERN and INFN [8,9]. This development led to the creation of the CERN spin-off company ADAM SA and the recent construction of LIGHT (Linac for Image Guided Hadron Therapy) as an industrial medical product [10]. Based on the LIBO and LIGHT structures, an IMPULSE linac has been designed to accelerate protons from $250 \mathrm{MeV}$ to $350 \mathrm{MeV}$ in less than $7 \mathrm{~m}$.

\section{A. The general linac layout and design method}

The IMPULSE linac is a Cell Coupled Linac (CCL). In this type of linacs, on-axis accelerating cells are magnetically coupled to off-axis coupling cells with a phase advance of $90 \mathrm{deg}$ between each successive cell. This makes the structure stable from the rf point of view. On the other hand, the beam acceleration process is performed in the $\pi$ mode allowing for maximum beam energy gain for a given input rf power [11].

The accelerator is divided into individual units powered by independent modulator/klystron systems. The power source are commercially available klystrons delivering $10 \mathrm{MW}$ peak power at the rf frequency of $2998.5 \mathrm{MHz}$ [12]. Each linac unit is divided in basic acceleration blocks called "tanks" (Fig. 2).

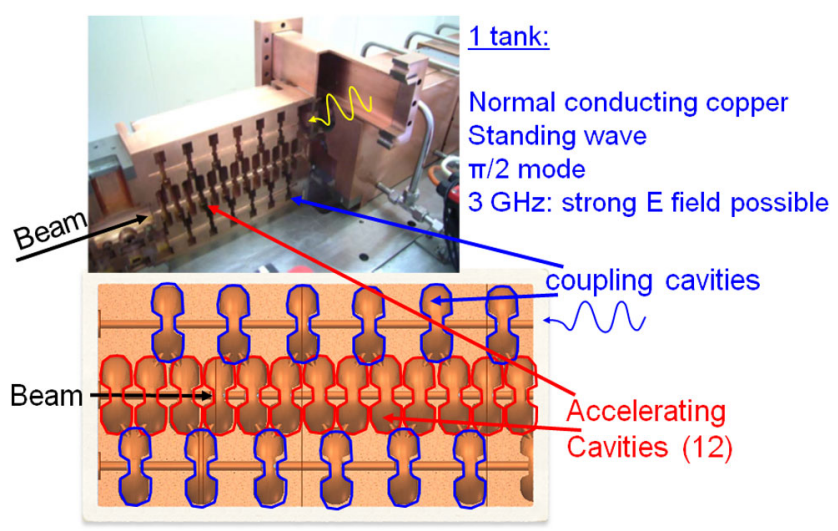

FIG. 2. Top: Picture of the LIBO module cut-away. Bottom: Schematic image of a CCL tank showing on-axis accelerating cells and off-axis coupling cells. 


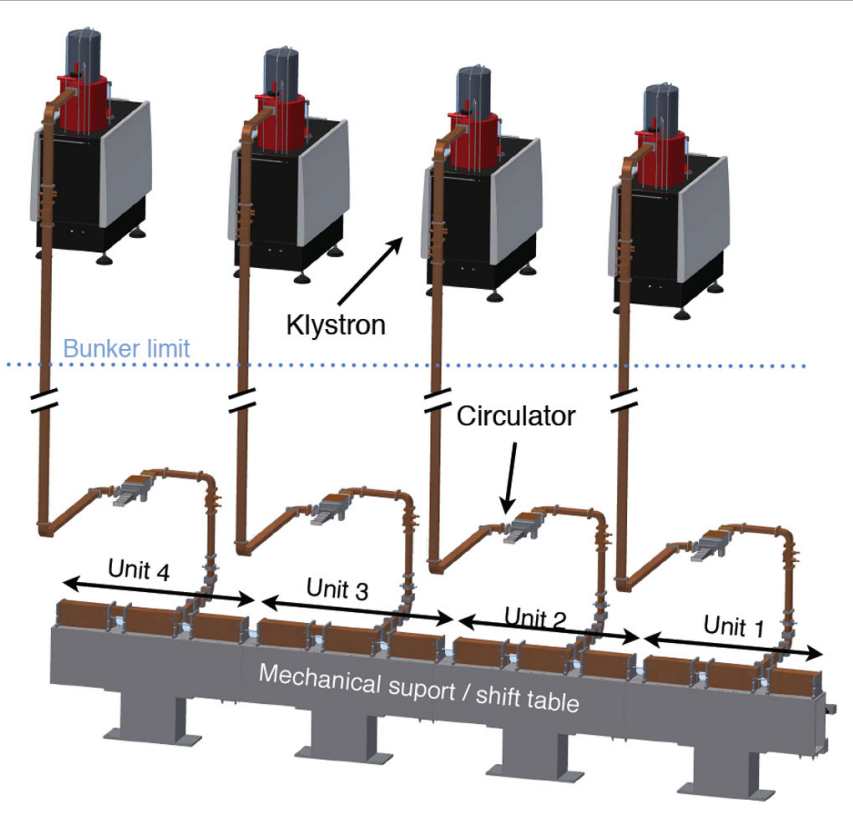

FIG. 3. Example of CCL linac booster.

Each tank contains several identical accelerating cells, which are driven via coupling cavities. The length of the accelerating cells in a given tank is constant, while for each tank the cavity length is increased to match in average the proton beam velocity (250 MeV: $0.61 c$ and $350 \mathrm{MeV}$ : $0.68 c$, where $c$ is the speed of light). The rf power is injected in the structure through a bridge coupler, which connects two or more consecutive tanks. A so-called unit consists of consecutive tanks, which are coupled to one rf klystron (Fig. 3).

On the sides of each tank, cooling plates are added to ensure the stability of the temperature during high power operation. All the above-mentioned components (accelerating cells, bridge coupler cells, waveguide, and cooling plates) are made of OFE Copper and are brazed together under vacuum in successive brazing steps. Between the consecutive tanks permanent magnetic quadrupoles (PMQs) focus the beam along the whole structure in a FODO-like lattice.

The linac layout design depends on a number of parameters, i.e., the number of tanks, number of cells per tank, free space between tanks, PMQ gradients and rf synchronous phase. The choice of the number of units, of tanks and of accelerating cells is first-of-all driven by the minimization of the number of needed power sources to achieve the desired energy gain. The number of cells per tank together with the number of tanks per unit is limited by the available power from the klystron and by the maximum electric field reached in the cells. In addition, rf mode overlapping and beam dynamics constraints must be considered. If the number of coupled cavities is too high there is the risk to excite modes with a phase advance different from $\pi / 2$, which introduces phase errors for the proton beam. In addition, increasing the number of cells, increases the tank length, which has a negative impact on the transversal beam dynamics, because focusing PMQs must act on a longer distance and counteract the defocusing effect of the electric fields in the accelerating gaps. Therefore, at this proton energy and rf frequency, the number of cells per tank should not exceed 18-20.

The synchronous phase $\phi_{s}$ with respect to the maximum of the rf field is another crucial parameter since it determines the energy gain of the structures and also the beam acceptance in the longitudinal plane (about $3 \phi_{s}$ for small $\phi_{s}$ ) [13]. More details on the linac design are reported in [7]. Since some energy spread is expected at the exit of the linac, the linac has to be followed by a set of bending magnets and an energy selecting slit.

\section{B. The rf design and beam dynamics studies}

Initial simulations were carried out with the code SUPERFISH [14], in order to optimize the accelerating cell geometry. The CCL geometry parameters were varied to define the set that maximizes the cavity efficiency (expressed in terms of the effective shunt impedance, $Z T^{2}$ ) without exceeding a surface electric field of $120 \mathrm{MV} / \mathrm{m}$, for safety operation below critical breakdown levels. Indeed, experimental results obtained in high gradient experiments conducted by TERA at CERN in collaboration with the CLIC group, show that even higher surface fields (up to $170 \mathrm{MV} / \mathrm{m}$ ) can be tolerated by wellconditioned cavities [15].

After the optimization of the accelerating cell geometry, the linac layout design was performed by successive iteration of the two codes DESIGN and LINAC [16]. The first one (DESIGN) calculates the length and power needed for each tank and additional information on the structure Twiss parameters. For the calculation of the required $\mathrm{rf}$ power, $30 \%$ power losses from the klystron to the tanks have been considered. This figure includes the usual losses in the waveguide network and in the bridge couplers.

The second code (LINAC) performs the tracking of multiparticle distributions through the linac structure and allows to evaluate transmission efficiency and beam acceptance. Indeed, a trade-off between transmission and linac length (or linac output energy) has to be found. For instance, the transmission of a beam of $350 \mathrm{MeV} \pm$ $5 \mathrm{MeV}$ has been studied for different apertures (bore radius) of the accelerating cells, as presented in Fig. 4. For these studies, the assumed geometrical emittances (99\%) of the beam at the output of the cyclotrons in the horizontal and vertical planes are respectively 6 and $10 \pi \mathrm{mm} \mathrm{mrad}$ [17]; a synchronous phase of $-25 \mathrm{deg}$ has been chosen (see next section). Orbit errors and misalignments of the structure were not considered in this preliminary study.

Figure 4 shows that the beam losses increase for bore radii smaller than $4.0 \mathrm{~mm}$, whilst they are almost constant for values above $4.0 \mathrm{~mm}$. This can be explained by the beam scraping for beam pipes smaller than $4.0 \mathrm{~mm}$. To 


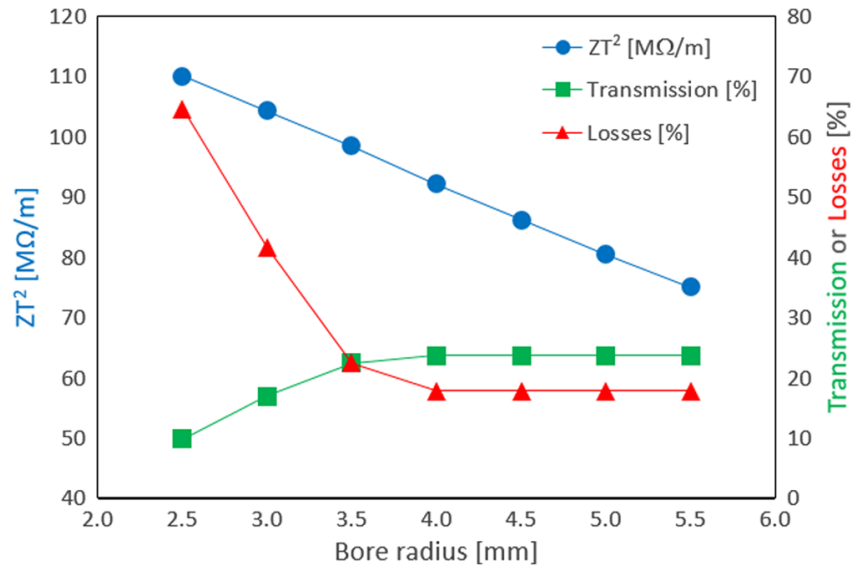

FIG. 4. Beam losses and beam transmission with respect to the bore radius [18]. The values of the effective shunt impedance for the first cell are also reported.

achieve maximal transmission, whilst maintaining an acceptable value of $Z T^{2}$ the bore radius has been chosen to be $4.0 \mathrm{~mm}$. The value of transmission in Fig. 4 refers to the fraction of the initial beam that is accelerated to the desired final energy of $350 \pm 5 \mathrm{MeV}$. The losses are the actual particles that are lost inside the linac. The remaining fraction consists of all the particles that are transmitted, but with lower energy. The linac will be followed by a system with sufficient dispersion by bending magnets. A slit system will then clean the beam and select the protons with the $350 \mathrm{MeV}$ energy of the linac.

All design choices are detailed in [18]. The final linac proposal is summarized in Table I.

TABLE I. Parameters table of IMPULSE linac booster.

\begin{tabular}{lc}
\hline \hline Parameter & Value \\
\hline Type of linac & CCL \\
rf frequency [MHz] & 2998.5 \\
Beam input-output energy [MeV] & $250-350$ \\
Total length [m] & 6.9 \\
Number of units & 4 \\
Cells per tank/tanks per unit & $13 / 3$ \\
Accelerating cell length (first-last) [mm] & $30.8-34.1$ \\
Bore hole diameter [mm] & 8.0 \\
Normalized transversal acceptance $[\pi \mathrm{mm}$ mrad] & 7.5 \\
Total number of PMQs & 13 \\
Length of each PMQ [mm] & 30 \\
PMQs gradients [T/m] & $210-220$ \\
rf synchronous phase [deg] & -25 \\
Peak power per module (with 30\% losses) [MW] & 7 \\
Effective Shunt Impedance ZT ${ }^{2}[\mathrm{M} \Omega / \mathrm{m}]$ & $94-99$ \\
Axial (average) electric field per cell [MV/m] & 25 \\
Klystron peak power [MW] & 10 \\
Klystron rf efficiency & 0.42 \\
Effective duration of rf pulses $[\mu \mathrm{s}]$ & 5 \\
Klystron maximum repetition rate $[\mathrm{Hz}]$ & 200 \\
rf duty cycle & $1 \times 10^{-3}$ \\
Plug power at 200 Hz [kW] & 120 \\
\hline \hline
\end{tabular}
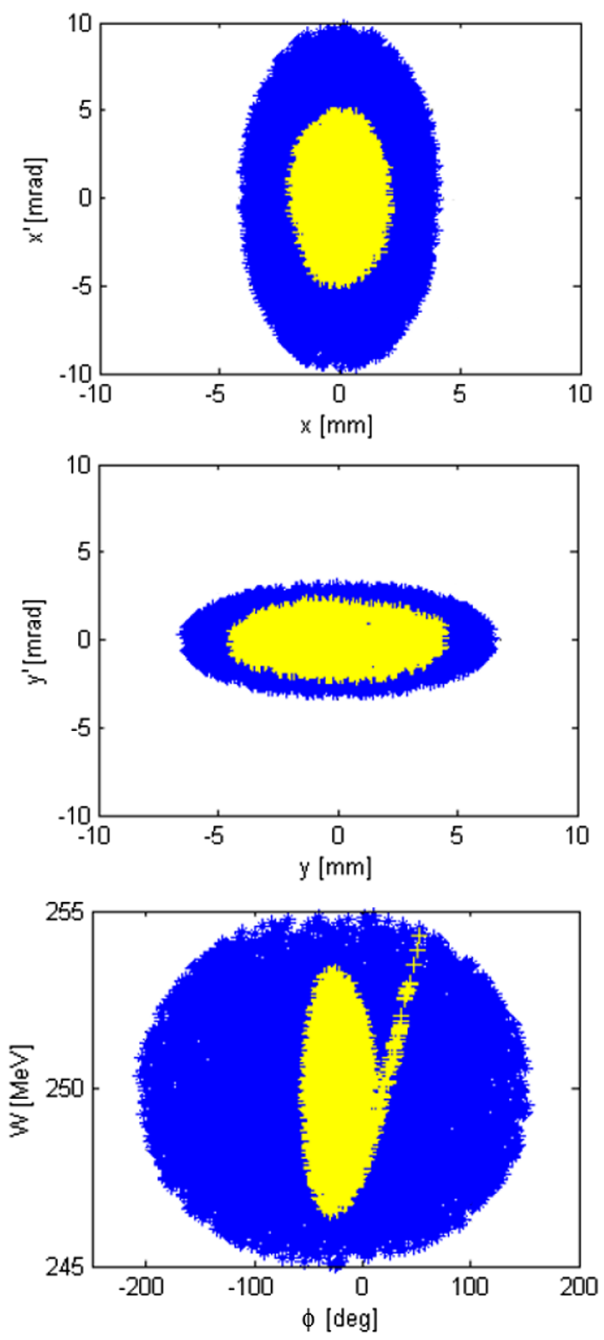

FIG. 5. Horizontal (top), vertical (middle), and longitudinal (bottom) acceptance of the linac [18]. The coordinates of the beam at the entrance of the first linac tank are plotted in the three phase spaces. In blue are the particles used as input beam, in yellow the particles that arrive to the end with the correct energy within $\pm 5 \mathrm{MeV}$.

The acceptance of the linac has been calculated in the transverse and longitudinal planes by analyzing the 6D coordinates at the entrance of the linac of the particles that reached the end with the right energy (within $\pm 5 \mathrm{MeV}$, corresponding to a $\pm 0.7 \%$ momentum spread, easily accepted by the downstream transfer line). The results of the simulations at $250 \mathrm{MeV}$ (shown in Fig. 5) give the following geometrical acceptances: (i) horizontal: $11.2 \pi \mathrm{mm} \mathrm{mrad}$ (ii) vertical: $10.7 \pi \mathrm{mm} \mathrm{mrad}$ (iii) longitudinal: $150 \pi$ deg MeV.

It should be noted that this geometrical acceptance has been calculated for beam particles arriving at the entrance of the linac booster at phase. Particles arriving out of phase will experience other field strengths so these will be accelerated to a different energy. The different focusing will also cause a change in the accelerated emittance. 
The particles that are off energy are still accepted by the permanent magnet lattice (i.e., are not lost inside the linac), but are then cleaned in a dispersive section of the transfer line after the linac.

\section{The cyclotron-linac coupling-longitudinal aspects}

The beam coming from the cyclotron is a continuous beam with a micro-structure bunched at a frequency of $72.23 \mathrm{MHz}$ (= two times the cyclotron rf frequency), corresponding to a bunch separation of approximately $14 \mathrm{~ns}$. The length of a particle bunch coming from the cyclotron is in the order of $0.8 \mathrm{~ns}$. This is still long compared to the rf buckets periodicity of the linac of $0.3 \mathrm{~ns}$. Therefore, during $0.8 \mathrm{~ns}$ and at the microscopic level, the input beam can be considered to be continuous from the linac dynamics perspective and this results in the cited small transmission through the linac. This is schematically represented in Fig. 6, where the linac rf periodicity is compared to the cyclotron bunches. The time structure in the cyclotron beam has been measured by means of a fast scintillation detector hit by the halo of the beam at PSI.

Since the linac rf system is pulsed at $200 \mathrm{~Hz}$ with a pulse length of only $5 \mu \mathrm{s}$ (Table I), the linac rf power is effectively off $99.9 \%$ of the time. During this off-time, the incoming beam is not accelerated. This, in combination with the fact that the energy of the incoming beam is $250 \mathrm{MeV}$, would lead to big activation of the cavities and radiation protection problems. The cyclotron frequency of $72 \mathrm{MHz}$ relates to bunch spacing, while $5 \mathrm{~ms}$ (the $200 \mathrm{~Hz}$ ) is the spacing of the linac rf pulses, each having a length of $5 \mu \mathrm{s}$. Thus the rf pulses cover $0.1 \%$ in time. During this pulse approximately $20 \%$ of the protons will be accepted in the rf of the linac. So the overall beam availability will be
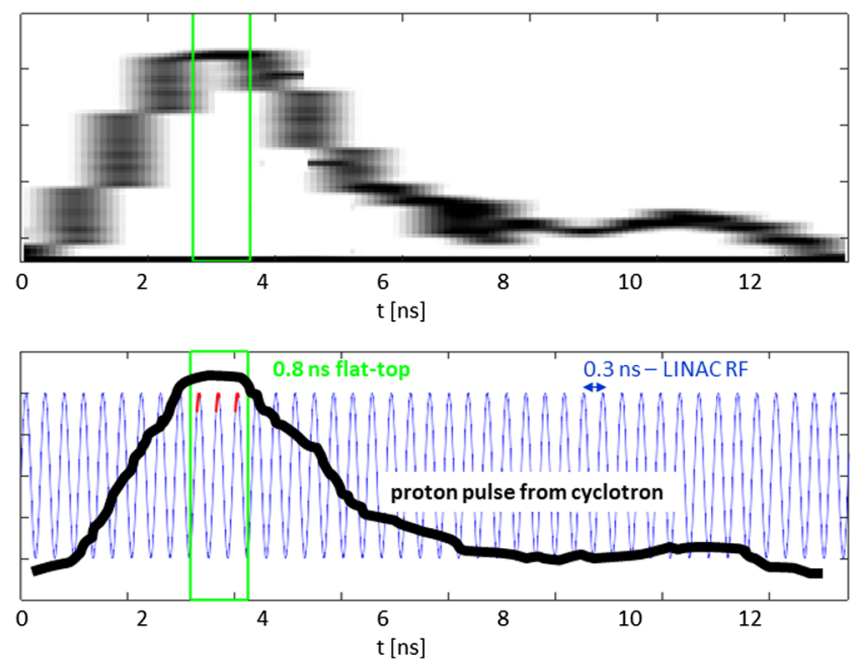

FIG. 6. Schematic representation of the linac longitudinal acceptance of a particle bunch coming from the cyclotron. About $20 \%$ of protons arrive at the right phase for acceleration in the linac as indicated by the section of sinusoids highlighted in red around the linac synchronous phase of $-25 \mathrm{deg}$. approximately $2 \times 10^{-4}$. In order to counteract the problems related to the different frequencies involved (schematically summarized in Fig. 7), the use of a beam chopping system in the cyclotron has been studied. The cyclotron beam is chopped in order to match the linac pulse repetition rate and furthermore it is possible to increase the beam intensity during these $5 \mu$ s pulses. So the few high-E pencil beams per treatment direction will hardly increase the treatment time and losses will be limited.

The chopping system consists of a pair of plates in the center of the cyclotron, that can deflect the beam in the vertical direction by means of an electric field. This vertical deflector is located in the central part of the cyclotron, because the beam energy is still in the order of a few hundred keV. A voltage difference on these plates will deflect this beam in a direction perpendicular to the acceleration plane, i.e., in the vertical direction. The beam is then partly intercepted by a vertical collimator covering the next few turns. In such a configuration, the amount of beam that is not intercepted by the collimator is dependent on the voltage applied to the plates. A voltage of $1.5 \mathrm{kV}$ is sufficient to stop the beam completely. When pulsing the beam intensity with a repetition rate of $200 \mathrm{~Hz}$ it is necessary that the rise/fall times of the deflecting voltage pulses are as short as possible, to minimize the amount of particles that will be lost in the booster.

In order to deliver this voltage with this frequency and short rise/fall times at the plates, one needs a power supply
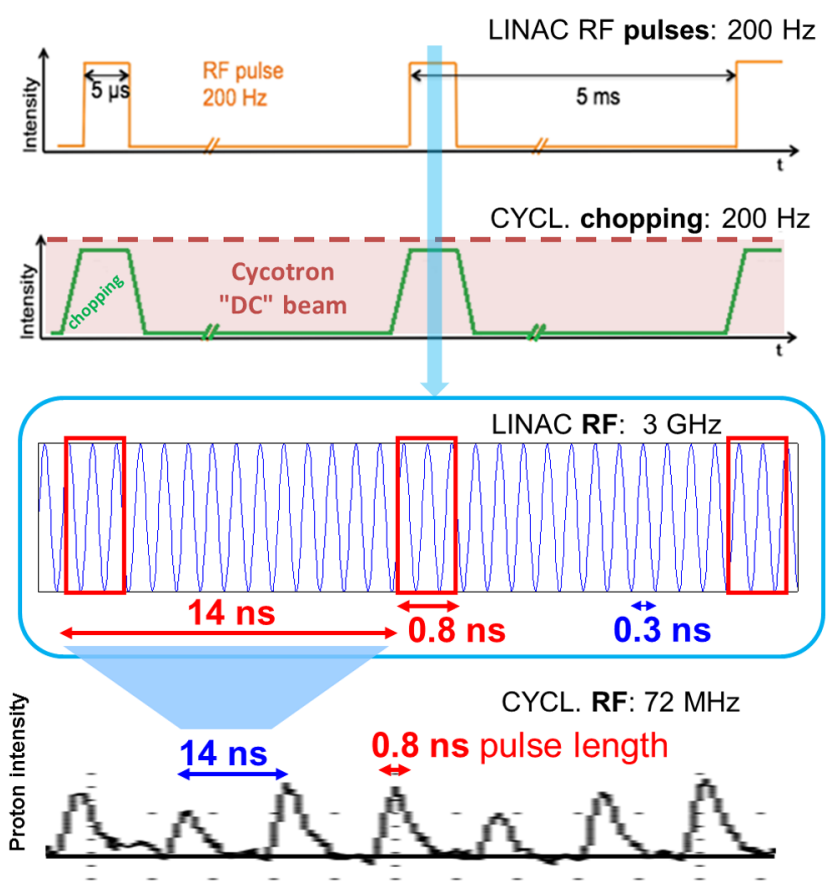

FIG. 7. Schematic time structure of linac and cyclotron. By chopping the cyclotron beam with the same repetition rate of the linac rf pulses $(200 \mathrm{~Hz})$ the losses are reduced. Within the cyclotron beam pulses (green line) a transmission of about $20 \%$ is expected. 

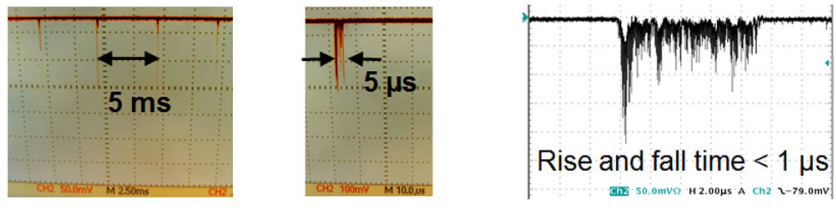

FIG. 8. Beam pulse intensity measured at PSI with a plastic scintillator. Left and middle: The beam pulse structure $(200 \mathrm{~Hz}$, $5 \mu \mathrm{s}$ ) is shown. Right: A rise/fall time of less than $1 \mu$ s could be achieved by mounting the existing power supply immediately next to the cyclotron.

with dedicated specifications of the output stage and one should use short low capacity cables. The capacity of the plates themselves can be neglected in this respect.

At PSI, a test has been done to investigate what rise times and frequencies can be achieved using the power supply normally employed to regulate the beam intensity in proton therapy. By detecting the beam intensity with a plastic scintillation detector, the beam intensity could be measured with a subnanosecond time resolution. Using the existing power supply, but mounted immediately next to the cyclotron, so that the total cable length was in the order of $5 \mathrm{~m}$, beam pulses with a length down to $5 \mu$ s could be observed, having a rise/fall time of $0.25 \mu \mathrm{s}$ (see Fig. 8). This is sufficiently short for the purpose. It is worth remarking that, with the normal cable length of almost $40 \mathrm{~m}$, a rise/fall time of $1.3 \mu \mathrm{s}$ was obtained [19].

\section{THE FIRST UNIT PROTOTYPE}

One of the advantages of the present linac solution is its modularity. A prototype of the first unit of the linac can indeed be built and could be tested in one of the existing PSI PROSCAN beam lines without considerable infrastructure modifications.

The IMPULSE prototype could consist of one unit of the booster, consisting of three tanks with a total length of $1.6 \mathrm{~m}$. The prototype unit should be placed in a straight section of the common beam line. To measure the energy spectrum behind the test unit, a bending magnet followed by a dispersive focus position should be mounted at some location behind the test unit. To measure the emittance behind the test unit, a quadrupole system followed by a profile monitor should be installed between the linac and the bending magnet. The method of measuring the width of the beam profile as a function of a quadrupole setting can be used to determine the beam emittance. The optimal location of the test unit within an existing proton therapy facility should be chosen such that there is a minimal interference with the ongoing treatment operation. To avoid such interference, we have proposed to make the design such that the prototype is placed on a movable table on a rail system, so that it can be moved in and out of the line within reasonable time.

Beam measurements performed at a possible test unit location at the PSI proton therapy facility gave a value for the transverse normalized emittance (4 rms) of $5.6 \pm$ $0.6 \pi \mathrm{mm} \mathrm{mrad}$ on the horizontal plane and of $3.4 \pm$ $1.0 \pi \mathrm{mm}$ mrad on the vertical plane, values that are smaller than the acceptance of the linac (see Sec. 3.3 of [18]). The expected output energy of the first unit is of $275 \mathrm{MeV}$ and can be analyzed with the beam monitors along the experimental line and by a small possible increase of the current of the following bending magnet.

The implementation of a test unit has been investigated for the situation in the proton therapy facility at PSI in 2011. However, such a test setup could also be installed in another facility, if its layout matches the needs listed in this section. Recently an approach similar to one studied for PSI has been proposed and is now been pursued by the University of Manchester and University of Lancaster with the PROBE project [20].

\section{CONCLUSION}

The upgrade of existing proton therapy facilities to higher beam energies ( $350 \mathrm{MeV}$ ) would allow us to address some open issues in proton therapy, enabling also the implementation of new specific treatment modalities with increased benefits for the patients. A high frequency linac is a modular and simple way of obtaining an energy boost of $100 \mathrm{MeV}$ with respect to the typical 230-250 MeV beams from a therapy cyclotron. For the PSI IMPULSE project, a $3 \mathrm{GHz}$ linac solution has been presented in this paper. The possibility to build a first unit prototype which could be mounted in an existing beam line of a therapy cyclotron, has also been discussed.

[1] T. Kanai, K. Kawachi, Y. Kumamoto, H. Ogawa, T. Yamada, H. Matsuzawa, and T. Inada, Spot scanning system for proton radiotherapy, Med. Phys. 7, 365 (1980).

[2] E. Pedroni, R. Bacher, H. Blattmann, T. Böhringer, A. Coray, A. Lomax, S. Lin, G. Munkel, S. Scheib, U. Schneider, A. Tourovsky, The 200-MeV proton therapy project at the Paul Scherrer Institute: Conceptual design and practical realization, Med. Phys. 22, 37 (1995).

[3] N. K. Abrosimov, Y. A Gavrikov, E. M. Ivanov, D. L. Karlin, A. V. Khanzadeev, N. N. Yalynych, G. A. Riabov, D. M. Seliverstov, and V. M. Vinogradov, $1000 \mathrm{MeV}$ proton beam therapy facility at Petersburg Nuclear Physics Institute Synchrocyclotron, J. Phys. Conf. Ser. 41, 424 (2006).

[4] J. Schippers and A. Lomax, Emerging technologies in proton therapy, Acta Oncologica 50, 838 (2011).

[5] R. Mohan, I. J. Das, and C. C. Ling, Empowering intensity modulated proton therapy through physics and technology: An overview, Int. J. Radiat. Oncol. Biol. Phys. 99, 304 (2017).

[6] J. Schippers et al., Next step in proton therapy: Boosting to $350 \mathrm{MeV}$ for therapy and radiography applications, in Proceedings of PTCOG 51 Conference, 2012, Seoul, http://www.ptcog.ch/index.php/past-programs-and-talks. 
[7] U. Amaldi, S. Braccini, and P. Puggioni, High frequency linacs for hadrontherapy, Rev. Accel. Sci. Techol. (RAST) 02, 111 (2009).

[8] U. Amaldi et al., LIBO - a linac-booster for protontherapy: Construction and tests of a prototype, Nucl. Instrum. Methods Phys. Res., Sect. A 521, 512 (2004).

[9] C. De Martinis et al., Acceleration tests of a $3 \mathrm{GHz}$ proton linear accelerator (LIBO) for hadrontherapy, Nucl. Instrum. Methods Phys. Res., Sect. A 681, 10 (2012).

[10] A. Degiovanni, D. Ungaro, and P. Stabile, LIGHT: A linear accelerator for proton therapy, in Proc. Of North America Particle Accelerator Conference, NAPAC16 (JACoW, Geneva, Switzerland, 2016).

[11] E. A. Knapp, B. C. Knapp, and J. M. Potter, Standing wave high energy linear accelerator structures, Rev. Sci. Instrum. 39, 979 (1968).

[12] Thales. Portfolio. https://www.thalesgroup.com/sites/default/ files/asset/document/high-power-klystrons.pdf, 2016.

[13] T. P. Wangler, Principles of RF Linear Accelerators (Wiley, New York, USA, 1998).

[14] Los Alamos Accelerator Code Group. Reference Manual for the POISSON/SUPERFISH Group of Codes, 1987.
[15] A. Degiovanni, R. Bonomi, M. Garlasché, S. VerdúAndrés, R. Wegner, and U. Amaldi, High gradient rf test results of S-band and C-band cavities, Nucl. Instrum. Methods Phys. Res., Sect. A 890, 1 (2018).

[16] K. Crandall, Documentation for program DESIGN and LINAC, 2006.

[17] J. Schippers, R. Dölling, J. Duppich, G. Goitein, M. Jermann, A. Mezger, E. Pedroni, H. W. Reist, and V. Vrankovic, The SC cyclotron and beam lines of PSI's new protontherapy facility PROSCAN, Nucl. Instrum. Methods Phys. Res., Sect. B 261, 773 (2007).

[18] J. Bilbao de Mendizabal, $3 \mathrm{GHz}$ linac booster design from $250 \mathrm{MeV}$ to $350 \mathrm{MeV}$ for medical application at PSI, Master's thesis, Université de Genève (2012).

[19] C. van Herwaarden, Improvements for the inner region of the COMET cyclotron, PSI Technical report, 2012.

[20] R. Apsimon, G. Burt, S. Pitman, and H. Owen, ProBEProton Boosting Extension for imaging and therapy, in Proceedings of International Particle Accelerator Conference, IPAC16 (JACoW, Geneva, Switzerland, 2016). 